\title{
Budget impact analysis of antiretroviral less drug regimen simplification in HIV-positive patients on the Italian National Health Service
}

This article was published in the following Dove Press journal:

ClinicoEconomics and Outcomes Research

23 September 2014

Number of times this article has been viewed

\author{
Umberto Restelli ${ }^{1,2}$ \\ Massimo Andreoni ${ }^{3}$ \\ Andrea Antinori ${ }^{4}$ \\ Marzia Bonfanti ${ }^{2}$ \\ Giovanni Di Perri ${ }^{5}$ \\ Massimo Galli ${ }^{6}$ \\ Adriano Lazzarin ${ }^{7}$ \\ Giuliano Rizzardini8,9 \\ Davide Croce ${ }^{1,2}$
}

'Department of Community Health, Faculty of Health Sciences, University of the Witwatersrand, Johannesburg,

South Africa; ${ }^{2}$ Centro di Ricerca in

Economia e Management in Sanità e nel Sociale (CREMS), Università Carlo Cattaneo - LIUC, Castellanza (VA), Italy; ${ }^{3}$ Clinical Infectious Diseases, Tor Vergata University (PTV), Rome, Italy; ${ }^{4}$ Clinical Department, National Institute for Infectious Diseases “L. Spallanzani,” Rome, Italy; ${ }^{5}$ Department of Medical Sciences, Infectious Diseases, Amedeo di Savoia Hospital, Turin, Italy; 'Third Division of Infectious Diseases, "Luigi Sacco" Hospital, Milan, Italy; 'Department of Infectious Diseases, San Raffaele Scientific Institute, Milan, Italy; ${ }^{8}$ First and Second Divisions of Infectious Diseases, "Luigi Sacco" Hospital, Milan, Italy; 'School of Clinical Medicine, Faculty of Health Sciences, University of the Witwatersrand, Johannesburg, South Africa
Correspondence: Umberto Restelli

Via San Michele, 14 - 21052,

Busto Arsizio (VA), Italy

Tel +3933 I930 724I

Fax +390331572513

Email urestelli@gmail.com
Background: Deintensification and less drug regimen (LDR) antiretroviral therapy (ART) strategies have proved to be effective in terms of maintaining viral suppression in human immunodeficiency virus (HIV)-positive patients, increasing tolerability, and reducing toxicity of antiretroviral drugs administered to patients. However, the economic impact of these strategies have not been widely investigated. The aim of the study is to evaluate the economic impact that ART LDR could have on the Italian National Health Service (INHS) budget.

Methods: A budget impact model was structured to assess the potential savings for the INHS by the use of ART LDR for HIV-positive patients with a 3 year perspective. Data concerning ART cost, patient distribution within different ARTs, and probabilities for patients to change ART on a yearly basis were collected within four Italian infectious diseases departments, providing ART to $13.7 \%$ of the total number of patients receiving ART in Italy.

Results: The LDR investigated (protease inhibitor-based dual and monotherapies) led to savings for the hospitals involved when compared to the "do nothing" scenario on a 3 year basis, between 6.7\% (23.11 million $€$ ) and 12.8\% (44.32 million $€$ ) of the total ART expenditures. The mean yearly cost per patient is reduced from $9,875 €$ in the do nothing scenario to a range between $9,218 €$ and $8,615 €$. The use of these strategies within the four departments involved would have led to a reduction of ART expenditures for the INHS of between $1.1 \%$ and $2.1 \%$ in 3 years.

Conclusion: ART LDR simplification would have a significant impact in the reduction of ART-related costs within the hospitals involved in the study. These strategies could therefore be addressed as a sustainable answer to the public financing reduction observed within the INHS in the last year, allowing therapies to be dispensed without affecting the quality of the services provided.

Keywords: antiretroviral therapy, Italy, budget impact model, monotherapy, dual therapy, cost

\section{Introduction}

Progress in human immunodeficiency virus (HIV) treatment has resulted in up to nearly $80 \%$ of patients reaching viral suppression (ie, HIV-1 RNA $<200$ copies $/ \mathrm{mL}$ ), with reduction of mortality and morbidity of HIV. ${ }^{1}$ The increase of virological success is reflected in an improvement in clinical and immunological outcomes.

Once the goal of achieving undetectable HIV-1 RNA has been accomplished, most patients will likely continue treatment with the same antiretroviral regimen for many years, without any need for change. However, for some patients, there are often important, nonvirologic reasons to modify their antiretroviral regimen, such as for drug intolerance or the need to simplify the regimen..$^{2-4}$ The perspective of long-term therapy and the availability of a high number of drugs with different toxicities and manageability 
favors a tailor-made therapeutic course, aimed to ensure consistent virological suppression and also improve quality of life for the patients. In Italian guidelines, this strategy is called "antiretroviral therapy (ART) optimization."

There are multiple potential reasons for considering a modification to the antiretroviral regimen of a virologically suppressed patient, including tolerability and toxicity ${ }^{5}$ and/or to preserve treatment options, avoid nucleoside reverse transcriptase inhibitor (NRTI) toxicity, or even decrease treatment cost. Two main reasons to change antiretroviral treatment could be considered: reduction of number of antiretroviral drugs (the so called simplification or "deintensification," leading to dual or monotherapy strategies) and reduction of daily dose, administration, and pill number (in a triple therapy strategy).

Physicians should discuss and evaluate with patients the potential risk/benefit ratio when establishing a new drug regimen to avoid long-term toxicities (preemptive switch) or drug interactions due to the need to treat other infections (tuberculosis, hepatitis B virus, hepatitis C virus, etc), to improve treatment adherence, to adjust for a planned pregnancy, or to accommodate a patient's request.

When modification is necessary, the first goal, as stated above, is to maintain viral suppression. Maintaining an undetectable HIV-1 RNA is certainly possible with most triple drug antiretroviral combinations in patients who do not harbor drug-resistant HIV strains.

Several studies have investigated the clinical efficacy of protease inhibitor (PI)-based monotherapies. ${ }^{6-10}$

Arribas et $\mathrm{al}^{6}$ compared monotherapy with darunavir $(\mathrm{DRV})+$ ritonavir (r) versus DRV/r plus two NRTIs in 256 patients with viral load $<50 \mathrm{HIV}-1$ RNA copies/mL, showing monotherapy's efficacy in being noninferior to the aforementioned triple therapy in a strict intention to treat analysis.

In a recent study, ${ }^{7} \mathrm{DRV}+\mathrm{r}$ monotherapy was compared with a DRV + r triple therapy strategy to maintain HIV-1 viral load suppression through a prospective, open-label, noninferiority, randomized, 96-week trial. DRV + r monotherapy was assessed as durable and efficacious for maintaining virological suppression in HIV-1 patients.

Moreover the efficacy of r-boosted PI monotherapies was assessed through systematic review, ${ }^{8}$ identifying 22 studies. Through this review, the authors suggest that "the majority of patients with prolonged viral suppression on highly active ART (HAART) can successfully be treated with PI monotherapy."

The European AIDS Clinical Society guidelines recognize r-boosted PI monotherapy with once-daily
$\mathrm{DRV}+\mathrm{r}$ or twice-daily lopinavir (LPV)/r as possible options in patients who have intolerance to NRTI, or for treatment simplification.

Pulido et $\mathrm{al}^{9}$ compared the 48-week efficacy of LPV/r monotherapy with LPV/r plus two nucleosides in 205 patients with suppressed HIV replication in a randomized, open-label, investigator-initiated, noninferiority, multicenter clinical trial. The results showed LPV/r monotherapy with reintroduction of nucleosides as needed to be noninferior to LPV/r plus two nucleosides.

A recent study published by d'Arminio Monforte et al ${ }^{10}$ confirmed in daily practice the efficacy and durability of $\mathrm{LPV} / \mathrm{r}$ monotherapy in 228 patients with at least two consecutive HIV RNA $\leq 50$ copies/mL in regimens including three or more drugs.

A further study published in $2012^{11}$ confirms the clinical efficacy of PI-based monotherapies, retrospectively analyzing 43 HIV-positive individuals who switched from a HAART regimen to either an atazanavir (ATV) + $r$ or an ATV monotherapy while virologically suppressed for at least 6 months to evaluate the cumulative proportion of virological failures and the immunologic variation. The authors stated that the study results "may support a potential role for ATV in PI monotherapy simplification strategies and encourage further studies on this topic." Despite these encouraging results, the use of ATV and ATV + r monotherapy has been challenged in different studies. ${ }^{12,13}$

In May 2013, the Italian Drug Agency approved the reimbursement of off-label monotherapy of DRV $+\mathrm{r}$ and LPV/r for HIV treatment, as per Italian law 648/96, based on the established use in clinical practice and scientific evidence. This agency decision is aligned with the most recent national guidelines ${ }^{14}$ concerning the use of antiretroviral drugs, recognizing the effectiveness of monotherapy.

The efficacy of dual therapy has been less investigated; however, available data foster encouragement for further investigations. Casado et $\mathrm{al}^{15}$ performed a retrospective cohort study on 44 HIV-infected patients on suppressive triple, $\mathrm{PI} / \mathrm{r}$-based HAART, who discontinued one nucleoside analog due to toxicity and continued dual therapy with lamivudine (3TC) plus the PI/r. At baseline, patients had viral suppression for a median time of 794 days, while receiving DRV $+r$ in 25 cases, LPV/r in 14 cases, and ATV $+r$ in five cases. The authors concluded that dual therapy with $3 \mathrm{TC}$ plus a boosted PI is a safe and effective simplification strategy in patients with toxicity to nucleoside analog.

In light of the results of the aforementioned study, the use of PI-based dual therapies with $3 \mathrm{TC}$ is considered a 
feasible simplification strategy in the clinical pathway for HIV/acquired immunodeficiency syndrome (AIDS) in the Lazio region. ${ }^{16}$

Furthermore, the AtLaS study ${ }^{17}$ investigated the 48-week safety and efficacy of the simplification to ATV $+r+3 T C$ in $40 \mathrm{HIV}$-positive patients on a stable ATV $+\mathrm{r}$ triple regimen, with viral load $<50 \mathrm{HIV}-1$ RNA copies/mL, CD4 count $>200$ cells $/ \mathrm{mm}^{3}$, and without previous treatment failure. The study results led the authors to state that this simplification strategy was "apparently safe and associated with rare virological failure, without resistance selection." However, the authors deem further investigation to be necessary.

Despite this evidence, the economic impact of simplification ART strategies (PI-based dual therapies and PI-based monotherapies) from the payer point of view has not been properly investigated. The aim of the study presented in this article is to evaluate the economic impact that ART less drug regimens (LDRs) could have on the Italian National Health Service (INHS) budget.

\section{Material and methods}

A budget impact model was structured to assess the potential savings for the INHS in the use of ART LDRs for HIVpositive patients with a 3 -year perspective. This time horizon was chosen as suggested by budget impact international guidelines. $^{18}$

The model was used to simulate five different scenarios: one that does not take into consideration any therapy simplification and four that consider different LDR simplification strategies (each of the last four scenarios was compared with the "do nothing" scenario to assess the differential cost for ART). LDRs were selected by the directors of six infectious diseases departments/wards of five Italian hospitals located in Milan, Rome, and Turin.

The total number of patients treated with ART was identified in the four hospitals involved (San Raffaele Hospital Specialist Clinic, "Luigi Sacco" Hospital, The National Institute for Infectious Disease "L Spallanzani" Specialist Clinic, Policlinico Tor Vergata) on December 31, 2012, and among them, the number of patients treated with PI-based treatments and with tenofovir (TDF)/emtricitabine (FTC) + efavirenz (EFV) or TDF/FTC/EFV. Further information on the number of patients following PI-based dual therapies and PI-based monotherapies was collected. The infectious diseases departments involved in the study in 2012 provided ART to $13.7 \%$ of the total number of patients receiving ART in Italy, as calculated by reprocessing data published by the Italian National Institute of Health. ${ }^{19}$
The model considers different therapies to be assigned to patients: triple therapies with PI, TDF/FTC + EFV or TDF/ FTC/EFV, PI-based monotherapies (LPV/r, ATV + r, or DRV $+\mathrm{r}$ ), and PI-based dual therapies (3TC + LPV/r, 3TC + DRV $+\mathrm{r}, 3 \mathrm{TC}+\mathrm{ATV}+\mathrm{r}$, or 3TC $+\mathrm{ATV})$. For dual therapies, only 3TC was considered as backbone since it was the only generic drug on the market in Italy at the time of the analysis, and in considering LDR simplification strategies, it could maximize the advantage for the INHS.

In year 0 , patients were assigned to different therapies as observed in the wards involved in the study. The distribution of patients within the therapies is reported in Table 1.

The hypotheses of the different LDR simplification scenarios are reported below.

\section{Scenario one}

Each cycle, one-third of the patients in PI-based triple therapy addressed as eligible for PI-based monotherapy and PI-based dual therapy are moved to PI-based monotherapy and PI-based dual therapy.

\section{Scenario two}

In the first cycle, all patients in PI-based triple therapy addressed as eligible for PI-based monotherapy and PI-based dual therapy are moved to PI-based monotherapy and PIbased dual therapy.

\section{Scenario three}

Each cycle, one-third of the patients in PI-based triple therapy and on TDF/FTC + EFV or TDF/FTC/EFV addressed as

Table I Patient distribution within the different therapeutic options in the model at time 0

\begin{tabular}{|c|c|}
\hline Total number of patients & 11,269 \\
\hline Patients treated with triple PI-based treatments & 4,763 \\
\hline Patients taking TDF/FTC + EFV or TDF/FTC/EFV & 2,014 \\
\hline $\begin{array}{l}\text { Patients taking } 3 T C+D R V+r 300 m g(I)+400 \mathrm{mg}(2) / / 00 \mathrm{mg} \\
\text { (I) }\end{array}$ & 52 \\
\hline $\begin{array}{l}\text { Patients taking } 3 T C+D R V+r 150 \mathrm{mg}(\mathrm{I})+600 \mathrm{mg}(\mathrm{I}) / 100 \mathrm{mg} \\
\text { (I) BID }\end{array}$ & 4 \\
\hline Patients taking 3TC + LPV/r 300 mg QD + 400 mg/ 100 mg BID & 68 \\
\hline Patients taking $3 \mathrm{TC}+\mathrm{ATV}+\mathrm{r} 300 \mathrm{mg}(\mathrm{I})+300 \mathrm{mg}(\mathrm{I}) / 100 \mathrm{mg}$ & 53 \\
\hline Patients taking 3TC + ATV $300 \mathrm{mg}$ QD + $400 \mathrm{mg}$ QD & 33 \\
\hline Patients taking DRV + r $400 \mathrm{mg}(2)+100 \mathrm{mg}(1)$ & 221 \\
\hline Patients taking DRV + r $600 \mathrm{mg}(1 \times 2)+100 \mathrm{mg}(I \times 2)$ & 8 \\
\hline Patients taking LPV/r $400 \mathrm{mg} / 100 \mathrm{mg}$ BID & 157 \\
\hline Patients taking ATV + r $300 \mathrm{mg}(\mathrm{I})+100 \mathrm{mg}(\mathrm{I})$ & 103 \\
\hline Patients taking other treatments & 3,793 \\
\hline
\end{tabular}

Note: Numbers in parentheses refer to the number of tablets per day. Abbreviations: 3TC, lamivudine; ATV, atazanavir; BID, twice a day; DRV, darunavir; EFV, efavirenz; FTC, emtricitabine; LPV, lopinavir; PI, protease inhibitor; QD, once a day; $r$, ritonavir; TDF, tenofovir. 


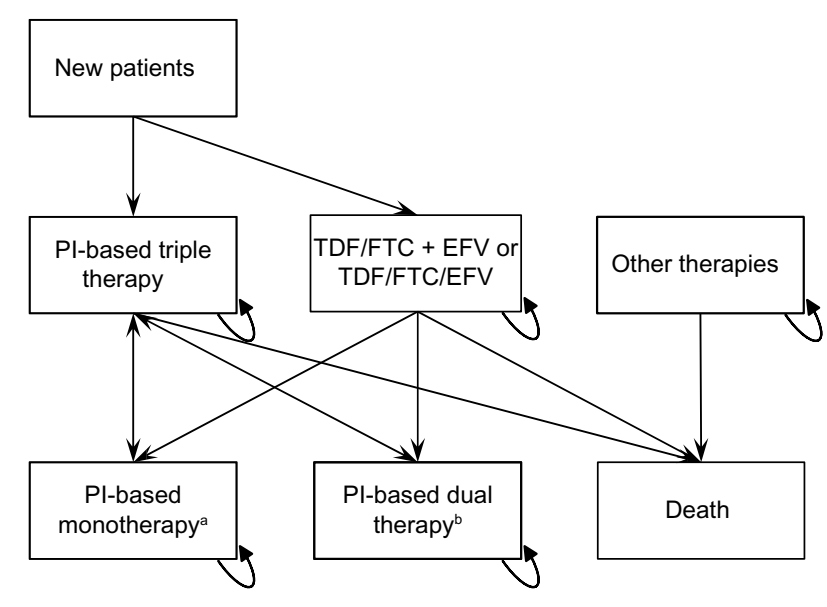

Figure I Structure of the budget impact model.

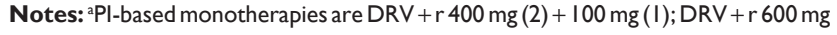
$(\mathrm{I} \times 2)+100 \mathrm{mg}(\mathrm{I} \times 2) ; \mathrm{LPV} / \mathrm{r} 400 \mathrm{mg} / 100 \mathrm{mg}$ BID; ATV + r $300 \mathrm{mg}(\mathrm{I})+100 \mathrm{mg}(\mathrm{I})$; bPI-based dual therapies are 3TC + DRV + r $300 \mathrm{mg}(\mathrm{I})+400 \mathrm{mg}(2) / 100 \mathrm{mg}(\mathrm{I})$; $3 T C+D R V+r 150 \mathrm{mg}(\mathrm{I})+600 \mathrm{mg}(\mathrm{I}) / 100 \mathrm{mg}$ (I) BID; 3TC + LPV/r $300 \mathrm{mg}$ $\mathrm{QD}+400 \mathrm{mg} / 100 \mathrm{mg}$ BID; 3TC + ATV + r $300 \mathrm{mg}(\mathrm{I})+300 \mathrm{mg}(\mathrm{I}) / 100 \mathrm{mg} ; 3 \mathrm{TC}+$ ATV $300 \mathrm{mg}$ QD $+400 \mathrm{mg}$ QD. Arrows represent the possible therapy changes for patients in every yearly cycle.

Abbreviations: 3TC, lamivudine; ATV, atazanavir; BID, twice a day; DRV, darunavir; EFV, efavirenz; FTC, emtricitabine; LPV, lopinavir; PI, protease inhibitor; QD, once a day; $r$, ritonavir; TDF, tenofovir.

eligible for PI-based monotherapy and PI-based dual therapy are moved to PI-based monotherapy and PI-based dual therapy.

\section{Scenario four}

In the first cycle, all patients in PI-based triple therapy and on TDF/FTC + EFV or TDF/FTC/EFV addressed as eligible for PI-based monotherapy and PI-based dual therapy are moved to PI-based monotherapy and PI-based dual therapy.

The structure of the model is presented in Figure 1.

In each cycle, a number of patients equal to $3 \%$ of the total number of patients of the previous cycle enter the model $(1.5 \%$ in PI-based triple therapy and $1.5 \%$ in TDF/FTC + EFV or $\mathrm{TDF} / \mathrm{FTC} / \mathrm{EFV}$ ), and $1 \%$ of the total number of patients of

Table 2 Percentage probability for patients using monotherapies and dual therapies to continue to use the same therapy in the following cycle, as assessed from data collected in the period January I, 2012-December 3I, 2012 in the wards involved in the analysis

\begin{tabular}{ll}
\hline Therapy & $\begin{array}{l}\text { Patients still using the } \\
\text { same therapy after I year }\end{array}$ \\
\hline $3 T C+$ DRV $+r$ & $91.9 \%$ \\
$3 T C+$ LPV/r & $90.5 \%$ \\
$3 T C+$ ATV $+r$ & $83.2 \%$ \\
DRV $+r$ & $92.1 \%$ \\
LPV/r & $74.1 \%$ \\
ATV $+r$ & $88.7 \%$
\end{tabular}

Abbreviations: 3TC, lamivudine; ATV, atazanavir; DRV, darunavir; LPV, lopinavir; $r$, ritonavir.
Table 3 Patient distribution within protease inhibitors-based triple therapies

\begin{tabular}{ll}
\hline Therapy & $\begin{array}{l}\text { Percentage } \\
\text { of patients }\end{array}$ \\
\hline TDF/FTC + ATV + r $(600 / 300 \mathrm{mg}+300 \mathrm{mg}+100 \mathrm{mg})$ & $48.7 \%$ \\
TDF/FTC + DRV + r $(600 / 300 \mathrm{mg}+800 \mathrm{mg}+100 \mathrm{mg})$ & $28.2 \%$ \\
TDF/FTC + LPV/r $(600 / 300 \mathrm{mg}+400 / 100 \mathrm{mg} \times 2)$ & $23.1 \%$ \\
\hline
\end{tabular}

Abbreviations: ATV, atazanavir; DRV, darunavir; FTC, emtricitabine; LPV, lopinavir; r, ritonavir; TDF, tenofovir.

the previous cycle exit the model due to death $(0.33 \%$ from PI-based triple therapy, $0.33 \%$ from TDF/FTC + EFV or $\mathrm{TDF} / \mathrm{FTC} / \mathrm{EFV}, 0.33 \%$ from other therapies).

Every cycle, patients could modify their therapy based on the hypothesis of each scenario or continue to use the same therapy as the previous cycle. The probability to change therapy was assessed by collecting data within the six wards involved and considering the number of patients in treatment with different therapies on January 1, 2012 and on December 31,2012. The percentage of patients still using the same therapy was considered the probability to continue to use the same therapy, not switching to PI-based triple therapy. The percentage related to monotherapies and dual therapies are presented in Table 2.

The clinical criteria to consider patients eligible for PI-based monotherapy were the following: HIV-RNA $<50$ copies $/ \mathrm{mL}$; CD 4 count $>200$ cells $/ \mathrm{mm}^{3}$; virologically controlled for at least 12 months; nadir $\mathrm{CD} 4$ count $>100$ cells $/ \mathrm{mm}^{3}$; no PI resistance mutations. Clinical criteria to consider patients eligible for PI-based dual therapy were: HIV-RNA $<50$ copies $/ \mathrm{mL}$; virologically controlled for at least 12 months; no PI resistance mutations.

Patients eligible for monotherapy were at the same time eligible for dual therapy; in this case, a monotherapy regimen was preferred.

The patient distribution in the three most administered PI-based triple therapies is reported in Table 3. The annual cost for this category of patients was considered to be the weighted mean of the three therapies.

Table 4 Total ART costs in year 0, divided by treatment typology

\begin{tabular}{lll}
\hline Therapy & Cost $(\boldsymbol{\epsilon})$ & $\%$ of total cost \\
\hline PI-based triple therapies & $46,399,663$ & $41.8 \%$ \\
PI-based dual therapies & $1,011,970$ & $0.9 \%$ \\
PI-based monotherapies & $2,191,971$ & $2.0 \%$ \\
TDF/FTC + EFV or TDF/FTC/EFV & $16,008,736$ & $14.4 \%$ \\
Other therapies & $45,476,44 \mathrm{I}$ & $40.9 \%$ \\
Total & $\mathrm{III}, 088,78 \mathrm{I}$ & $100 \%$
\end{tabular}

Abbreviations: ART, antiretroviral therapy; EFV, efavirenz; FTC, emtricitabine; PI, protease inhibitor;TDF, tenofovir. 
Table 5 Yearly and total ART cost and per capita ART cost for each scenario

\begin{tabular}{|c|c|c|c|c|c|c|c|c|}
\hline \multirow[t]{2}{*}{ Scenario } & \multicolumn{4}{|c|}{ Total ART cost to treat the sample (€) } & \multicolumn{4}{|c|}{ Per capita ART cost $(€)$} \\
\hline & Year I & Year 2 & Year 3 & 3 years total & Year I & Year 2 & Year 3 & 3 years mean \\
\hline Do nothing & $113,472,398$ & $115,800,466$ & $118,098,395$ & $347,37 \mid, 260$ & 9,872 & 9,877 & 9,876 & 9,875 \\
\hline Scenario one & $109,236,201$ & $107,932,762$ & $107,089,570$ & $324,258,533$ & 9,503 & 9,206 & 8,955 & 9,218 \\
\hline Scenario two & $100,763,807$ & $104,900,243$ & $108,677,786$ & $3|4,34|, 836$ & 8,766 & 8,947 & 9,088 & 8,936 \\
\hline Scenario three & $107,659,058$ & $105,122,653$ & $103,327,709$ & $316,109,420$ & 9,366 & 8,966 & 8,640 & 8,986 \\
\hline Scenario four & $96,032,378$ & $101,201,345$ & $105,822,532$ & $303,056,254$ & 8,355 & 8,632 & 8,849 & 8,615 \\
\hline
\end{tabular}

Abbreviation: ART, antiretroviral therapy.

The costs of ART refer to 2013 and are value added tax inclusive, as reported in the Lombardy Region HIV/AIDS Clinical Diagnostic Pathway. ${ }^{20}$

The cost of ART for each patient in the "other therapies" category was calculated by subtracting the costs related to PI-based therapies, TDF/FTC + EFV, and TDF/FTC/EFV from the costs of all the therapies administered to patients within the wards involved in the study, and dividing it by the number of patients treated in each ward not with the aforementioned therapies (PI-based therapies, TDF/FTC + $\mathrm{EFV}$, and TDF/FTC/EFV).

\section{Results}

The costs to treat the sample per treatment typology in year 0 are reported in Table 4, and the total cost to treat the sample and the cost per patient in each scenario are reported in Table 5. The difference in terms of 3-year cost between the do nothing scenario and the other scenarios are $-6.7 \%$ for scenario one, $-9.5 \%$ for scenario two, $-9.0 \%$ for scenario three, and $-12.8 \%$ for scenario four.

On a 3-year basis, the mean yearly cost per patient is reduced from $9,875 €$ in the do nothing scenario to $9,218 €$ in scenario one, $8,936 €$ in scenario two, $8,986 €$ in scenario three, and $8,615 €$ in scenario four.

The 3-year impact on the INHS of the LDR simplification strategies presented, considered as the difference between the "do nothing scenario" and each LDR simplification scenario, is between -23.11 million $€$ and -44.32 million $€$, with mean yearly savings between -7.70 million $€$ and -14.77 million $€$.

Considering the last available data on the total ART expenditure in Italy, 689.4 million $€$ in $2012,{ }^{21}$ the implementation of the LDR simplification strategies presented above within the hospitals involved in the study would lead to a reduction of the ART expenditures at a national level, considering the mean 3 -year reduction is between $-1.1 \%$ and $-2.1 \%$.

\section{Discussion}

ART LDR would have a significant impact in the reduction of ART-related costs within the hospitals involved in the study. Considering the encouraging results of the clinical studies presented in the background and the public financing reduction of the INHS, the need to identify strategies that could reduce the per capita cost to provide therapies not affecting the quality of the services provided could be addressed by ART LDR simplification strategies.

In the literature, this topic was addressed mainly through head to head cost effectiveness studies; a budget impact analysis taking the INHS point of view was lacking. The only such analysis in the literature is a 2014 study by Angeletti et al, ${ }^{22}$ whose objective, however, was the assessment of different cost saving strategies. Among these, the switch of $20 \%-40 \%$ of stable first and second line patients from PI-based triple therapies to PI-based monotherapies was estimated to lead to yearly savings for the Health Service of the Lazio region of between 1.0 million $€$ and 1.9 million $€$, considering the total cost for the Regional Health Service to provide care to patients (considering antiretroviral drugs, nonantiretroviral drugs, hospital admissions, out-patient care, and laboratory tests). These amounts represent $0.6 \%$ and $1.3 \%$, respectively, of the yearly regional cost of care of HIV-positive patients.

\section{Conclusion}

The analysis presented shows the potential savings that LDR simplification strategies could lead to for the INHS, if applied widely. The main strength of the analysis is related to the data considered, which reflect the real clinical practice of the wards involved. PI-based dual therapies and monotherapies were taken into consideration in the analysis, including the ones considered off-label (ATV $+r$ monotherapy) when detected within the hospitals. The main weakness of the model is that it does not take into consideration the costs of therapy switch, and considers only ART costs. Moreover, we assumed that all the patients eligible for a LDR simplification are switched to a dual therapy or monotherapy within the 3-year time horizon considered.

In light of the potential savings that emerged in the analysis, the switch of eligible patients receiving PI-based 
triple, $\mathrm{TDF} / \mathrm{FTC}+\mathrm{EFV}$, or TDF/FTC/EFV therapies should be regarded as a feasible and cost-saving strategy.

\section{Disclosure}

The authors report no conflicts of interest in this work.

\section{References}

1. Moore RD, Bartlett JG. Dramatic decline in the HIV-1 RNA level over calendar time in a large urban HIV practice. Clin Infect Dis. 2011;53(6): 600-604.

2. Reina E, San Miguel R, Larrea N, Garcia P, Napal V. Potential for simplification of HIV treatment with boosted protease inhibitor monotherapy. Int J Clin Pharm. 2012;34(6):911-916.

3. Ford N, Flexner C, Vella S, Ripin D, Vitoria M. Optimization and simplification of antiretroviral therapy for adults and children. Curr Opin HIV AIDS. 2013;8(6):591-599.

4. Llibre JM, Cardona G, Santos JR, et al. Antiretroviral treatment switch strategies for lowering the costs of antiretroviral therapy in subjects with suppressed HIV-1 viremia in Spain. Clinicoecon Outcomes Res. 2013;5:215-221.

5. Elzi L, Marzolini C, Furrer H, et al; Swiss HIV Cohort Study. Treatment modification in human immunodeficiency virus-infected individuals starting combination antiretroviral therapy between 2005 and 2008. Arch Intern Med. 2010;170(1):57-65.

6. Arribas JR, Clumeck N, Nelson M, Hill A, van Delft Y, MoecklinghoffC. The MONET trial: week 144 analysis of the efficacy of darunavir/ ritonavir (DRV/r) monotherapy versus DRV/r plus two nucleoside reverse transcriptase inhibitors, for patients with viral load $<50$ HIV-1 RNA copies/mL at baseline. HIV Med. 2012;13(7):398-405.

7. Valantin MA, Lambert-Niclot S, Flandre P, et al; MONOI ANRS 136 Study Group. Long-term efficacy of darunavir/ritonavir monotherapy in patients with HIV-1 viral suppression: week 96 results from the MONOI ANRS 136 study. J Antimicrob Chemother. 2012;67(3):691-695.

8. Bierman WF, van Agtmael MA, Nijhuis M, Danner SA, Boucher CA. HIV monotherapy with ritonavir-boosted protease inhibitors: a systematic review. AIDS. 2009;23(3):279-291.

9. Pulido F, Arribas JR, Delgado R, et al; OK04 Study Group. Lopinavir-ritonavir monotherapy versus lopinavir-ritonavir and two nucleosides for maintenance therapy of HIV. AIDS. 2008;22(2):F1-F9.

10. d'Arminio Monforte A, Gianotti N, Cozzi-Lepri A, et al; ICONA Foundation Cohort. Durability of lopinavir/ritonavir monotherapy in individuals with viral load $\leq 50$ copies $/ \mathrm{mL}$ in an observational setting. Antivir Ther. 2014;19(3):319-324.

11. Cossarini F, Salpietro S, Galli L, et al. Monotherapy with atazanavir as a simplification strategy: results from an observational study. $J$ Acquir Immune Defic Syndr. 2012;60(3):e101-e103.

12. Karlström O, Josephson F, Sönnerborg A. Early virologic rebound in a pilot trial of ritonavir-boosted atazanavir as maintenance monotherapy. J Acquir Immune Defic Syndr. 2007;44(4):417-422.
13. Castagna A, Spagnuolo V, Galli L, et al. 48-weeks outcomes of atazanavir/ritonavir monotherapy as maintenance strategy in HIV-1 treated subjects with viral suppression: interim analysis results of the MODAT Study. 14th European AIDS Conference; October 16-19, 2013; Brussels, Belgium. Abstract PS4/2. Available from: http://eacs.multilearning.com/eacs/2013/14th/39215/antonella.castagna.48-weeks.outcomes.of.atazanavir.ritonavir.monotherapy.as.html?history_id=689784. Accessed May 9, 2014.

14. Ministry of Health, Commissione Nazionale per la lotta contro l'AIDS, Consulta delle Associazioni per la lotta contro l'AIDS, Società Italiana di Malattie Infettive e Tropicali. Linee Guida Italiane sull'utilizzo dei farmaci antiretrovirali e sulla gestione diagnostico-clinica delle persone con infezione da HIV-1. Ministero della Salute; 2013. Italian. Available from: http://www.salute.gov.it/imgs/C_17_pubblicazioni_2074_ allegato.pdf. Accessed May 9, 2014.

15. Casado JL, de la Calle C, del Palacio M, Perez-Elías MJ, Moreno A, Moreno S. Short communication: lamivudine plus a boosted-protease inhibitor as simplification strategy in HIV-infected patients: proof of concept. AIDS Res Hum Retroviruses. 2013;29(3):588-591.

16. Decreto del commissario ad acta 13 gennaio 2014, n 2. Aggiornamento del decreto "Razionalizzazione dell'uso di farmaci per la terapia antiretrovirale HIV". Available from: http://www.regione.lazio.it/binary/ rl_sanita/tbl_normativa/Decr_U00002_13_1_13_aggiornamento_ terapia_HIV.pdf. Accessed May 9, 2014.

17. Di Giambenedetto S, Fabbiani M, Colafigli M, et al. Safety and feasibility of treatment simplification to atazanavir/ritonavir + lamivudine in HIV-infected patients on stable treatment with two nucleos(t)ide reverse transcriptase inhibitors + atazanavir/ritonavir with virological suppression (Atazanavir and Lamivudine for treatment Simplification, AtLaS pilot study). J Antimicrob Chemother. 2013;68(6):1364-1372.

18. Mauskopf JA, Sullivan SD, Annemans L, et al. Principles of good practice for budget impact analysis: report of the ISPOR Task Force on good research practices - budget impact analysis. Value Health. 2007;10(5):336-347.

19. Raimondo M, Camoni L, Regine V, et al. Caratteristiche delle persone che vivono con L'HIV e con L'AIDS in Italia [Characteristics of people living with HIV and AIDS in Italy]. Not Ist Super Sanita. 2013;26(7-8): 3-6. Italian.

20. Directorate General for Health of Lombardy Region D.d.g. March 1, 2013 - n 1725 - Approvazione del documento avente ad oggetto "Percorso diagnostico terapeutico (PDT) del paziente affetto da malattia HIV/AIDS. Available from: http://www.ao.lodi.it/infoglueDeliverLive/digitalAssets/102/102169_Percorso_diagnostico_terapeutico_del_paziente_affetto_da_malattia_HIV_AIDS_BURL_4_3_2013. sflb.pdf. Accessed August 1, 2014.

21. Agenzia Italiana del Farmaco. L'uso dei farmaci in Italia-Rapporto Nazionale Anno 2012. Roma; 2013. Available from: http://www. agenziafarmaco.gov.it/sites/default/files/Rapporto_OsMed_2012.pdf. Accessed May 9, 2014.

22. Angeletti C, Pezzotti P, Antinori A, et al. Antiretroviral treatment-based cost saving interventions may offset expenses for new patients and earlier treatment start. HIV Med. 2014;15(3):165-174.
ClinicoEconomics and Outcomes Research

\section{Publish your work in this journal}

ClinicoEconomics \& Outcomes Research is an international, peerreviewed open-access journal focusing on Health Technology Assessment, Pharmacoeconomics and Outcomes Research in the areas of diagnosis, medical devices, and clinical, surgical and pharmacological intervention. The economic impact of health policy and health systems

\section{Dovepress}

organization also constitute important areas of coverage. The manuscript management system is completely online and includes a very quick and fair peer-review system, which is all easy to use. Visit $\mathrm{http}: / /$ www.dovepress.com/testimonials.php to read real quotes from published authors. 